\title{
RNF8 plays an important role in the radioresistance of human nasopharyngeal cancer cells in vitro
}

\author{
MAOXIN WANG* ${ }^{*}$ XIANMING CHEN* ${ }^{*}$ HUI CHEN, XIAN ZHANG, JIANZHONG LI, \\ HONGXUN GONG, CHEN SHIYAN and FAN YANG \\ Fuzong Clinical College of Fujian Medical University, Fuzhou General Hospital \\ of Nanjing Command Zone of PLA, Fuzhou, Fujian, P.R. China
}

Received January 25, 2015; Accepted April 20, 2015

DOI: $10.3892 /$ or.2015.3958

\begin{abstract}
Tumor residue or recurrence is common after radiation therapy for nasopharyngeal cancer (NPC) since the tumor cells can repair irradiation-induced DNA damage. The ubiquitination cascade mediates the assembly of repair and signaling proteins at sites of DNA double-strand breaks (DSBs). Ring finger protein 8 (RNF8) is an E3 ubiquitin ligase that triggers ubiquitination at the site of DSBs. The present study aimed to identify whether and how RNF8 small interfering RNA (siRNA) treatment enhances the radiosensitivity of irradiated human NPC cell lines. The CNE1, CNE2, and SUNE human NPC cell lines were stably transfected with a constructed RNF8-targeting siRNA expression vector. Western blotting was used to detect the effectiveness of RNF8 downregulation by RNF8 siRNA. The siRNA-transfected (RNF8-) and non-transfected $\left(\mathrm{RNF}^{+}\right)$cells were irradiated at different doses by a linear accelerator. The growth inhibition ratio and apoptosis rate were detected by the methyl thiazolyl tetrazolium (MTT) assay and flow cytometry, respectively. The ataxia-telangiectasia mutated (ATM), DNA-PKcs, Chk1, Chk2, Nbs1 and Ku80 protein levels in each group were determined. The growth inhibition ratio and apoptotic percentage of RNF8- cells were higher than those of the RNF8+ cells in each of the three cell lines. Lower protein expression levels of Chk1, Chk2, ATM, and Nbs1 were observed in the irradiated RNF8- cells compared to the irradiated $\mathrm{RNF}^{+}$cells in each of the three cell lines $(\mathrm{P}<0.01)$. As a result, a conclusion could
\end{abstract}

Correspondence to: Dr Maoxin Wang, Fuzong Clinical College of Fujian Medical University, Fuzhou General Hospital of Nanjing Command Zone of PLA, 156 Xihuanbei Road, Fuzhou, Fujian, P.R. China

E-mail: entmao@126.com

${ }^{*}$ Contributed equally

Key words: ring finger protein 8, ubiquitination, E3 ubiquitin ligase, nasopharyngeal cancer cells, irradiation therapy, radioresistance, DNA double-strand breaks be drawn that RNF8 recruits and ubiquitinates many factors to repair DNA damage, including DSBs, thereby conferring radioresistance to NPC cells.

\section{Introduction}

Nasopharyngeal cancer (NPC) shows distinct racial and geographical distributions (1). A rare disease in Europe and USA, NPC is common in Southern China, with a peak incidence in this region approaching 30 cases per 100,000 individuals per year (2). The main therapy for NPC is radiation, and the 5-year overall survival rate of patients with NPC after radiation or multimodal therapy is approximately $75 \%(3-8)$. Although the effects of radiation therapy are reliable, many patients die of NPC recurrence and therapeutic failure due to radioresistance, which has become a serious problem and is receiving growing attention.

Radiation kills cancer cells by creating double-strand breaks (DSBs). However, if cancer cells become radioresistant, then they can repair the damaged DNA when DSBs occur. Ubiquitination and, more particularly, E3 ubiquitin ligases play important roles in the DNA damage response (9-12). One of the key E3 ubiquitin ligases in the response to DNA damage is ring finger protein 8 (RNF8), a member of the RING finger-containing nuclear factor family (13-15). RNF8 is a 485-residue nuclear polypeptide with an N-terminal forkhead-associated (FHA) domain and a C-terminal ring domain. RNF8 rapidly accumulates at DSBs via the interaction of its FHA domain. It recruits and ubiquitinates checkpoint proteins and other DNA repair proteins at the site of DNA damage, resulting in DNA repair $(13,16-19)$.

Previous studies have shown that RNF8 can ubiquitinate different proteins and repair irradiation-induced DNA damage, thereby promoting cellular resistance to radiation (11). There are two main mechanisms for repairing DSBs in DNA: nonhomologous end-joining (NHEJ) and homologous recombination (HR) $(20,21,26-29)$. The key enzymes in NHEJ and HR are members of the phosphoinositide 3-kinase (PI3K) family, namely DNA-dependent protein kinase (DNA-PK) (22-24) and ataxia-telangiectasia mutated (ATM), respectively. DNA-PK, which contains the DNA-PKcs subunit, is a critical component of the DNA repair machinery that governs the response to DNA damage, serving to main- 
tain genome integrity (25). In contrast, ATM regulates the cell cycle and repairs DNA breaks. After cell irradiation, ATM is directly ubiquitinated and recruited by RNF8 or activated by the Mre11-Rad50-Nbs1 (MRN) complex (9,11). ATM phosphorylates proteins, such as the cell-cycle regulation kinases Chk1 and Chk2, to trigger the checkpoint to repair DNA damage. In NHEJ, the repairing of DNA strands may come from different strands, whereas in HR, the strands must have a homologous sequence and pair according to base-pairing principles $(30,31)$. RNF8-deficient cells are defective in the G2/M checkpoint and exhibit sensitivity to ionizing radiation $(14,15,32)$.

In this study, we examined whether RNF8 causes DNA repair and promotes the radioresistance of NPC cells. The aim of this study was to examine whether and how RNF8-targeting small interfering RNAs (siRNAs) enhance the radiosensitivity of human NPC cells.

\section{Materials and methods}

Cell lines and cell culture. CNE1, CNE2 and SUNE NPC cell lines were purchased from the Institute of Biochemistry and Cell Biology, Shanghai Institutes for Biological Sciences, Chinese Academy of Sciences Cell Bank (Shanghai, China). Cells were maintained in RPMI-1640 medium containing $10 \%$ fetal calf serum (FCS; Hangzhou Sijiqing Biological Engineering Materials Co., Ltd., Hangzhou, China). Cells in the logarithmic phase of growth were used for all experiments.

Short hairpin RNAs (shRNAs) and transfection of plasmids. The shRNAs against human RNF8 and control shRNAs (noneffective short hairpin green fluorescent protein, shGFP) were designed and synthesized by Gene Pharma (Shanghai, China). The shRNA target sequence for RNF8 was GGACAAUUAU GGACAACAAG A, which was previously described (9). Cells were seeded at $1 \times 10^{5}$ cells/well in 6-well plates and cultured in antibiotic-free medium for $12 \mathrm{~h}$. When the cell confluency reached $30-50 \%$, the cells were transfected with the control and RNF8 shRNA plasmids by the Lipofectamine 2000 reagent (Invitrogen, Carlsbad, CA, USA), in accordance with the manufacturer's instructions. Six hours later, the cells were switched to RPMI-1640 medium containing 10\% FCS. After $24 \mathrm{~h}$, fluorescence microscopy was used to confirm the transfection efficiency. Transfection was repeated three times with an interval of $24 \mathrm{~h}$ to achieve the maximal effect.

Selection of stably transfected cells. After 2 days, the cells were cultured in medium containing the minimum concentration of hygromycin needed to kill all of the non-transfected cells while the transfected cells survived. Then, the cells were grown without antibiotic for 10-14 days, until they repopulated the culture vessel. These cells were considered to be stably transfected. The efficiency of stable transfection of the siRNAs was measured by western blot analysis.

Cell grouping and irradiation of the cells. Cells were divided into three coupled groups. Groups A1, B1 and $\mathrm{C} 1$ consisted of CNE1, CNE2 and SUNE cells exhibiting downregulated RNF8 gene expression, respectively. Groups A2, B2 and C2 were untreated CNE1, CNE2 and SUNE cells, respectively.

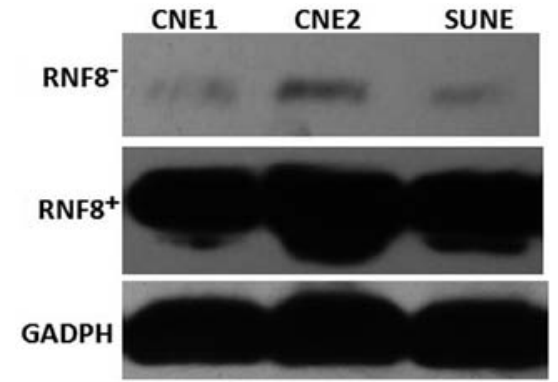

Figure 1. Western blot results of RNF8 expression in the CNE1, CNE2 and SUNE human nasopharyngeal cell lines that had been transfected (RNF8-) or not $\left(\mathrm{RNF}^{+}\right)$with RNF8-targeting siRNAs. GADPH was used as a loading control. RNF8, ring finger protein 8 .

A linear accelerator (BJ-6B; Yikeda Medical Instrument Co., Ltd., China) with a source skin distance of $75 \mathrm{~cm}$, radiation area of $20 \times 20 \mathrm{~cm}$ and dose rate of $464 \mathrm{cGy} / \mathrm{min}$ was used to deliver different doses of radiation $(0,4,6,8$ and $10 \mathrm{~Gy})$ to each group. The culture medium in each well or flask was immediately replaced with fresh medium for subsequent experiments.

Methyl thiazolyl tetrazolium (MTT) assay. MTT (MedChem Express, Princeton, NJ, USA) was dissolved in phosphate-buffered saline (PBS) and adjusted to a concentration of $5 \mathrm{mg} / \mathrm{ml}$. A total of 3,000 NPC cells were cultured per well in 96-well plates. After $36 \mathrm{~h}$ of culture, the cells were subjected to different doses of irradiation. After 12, 24, 36 and 48 h, $20 \mu \mathrm{l}$ of MTT was added to each well of the 96-well plate and incubated for $4 \mathrm{~h}$ at $37^{\circ} \mathrm{C}$. After incubation, the culture medium was removed, and $150 \mu \mathrm{l}$ of dimethyl sulfoxide was added to each well. The 96-well plates were gently swirled in the dark for $10 \mathrm{~min}$ at room temperature. Absorbance values at $490 \mathrm{~nm}$ $\left(\mathrm{A}_{490}\right)$ of each well were measured by an enzyme-linked immunosorbent detector (Model 550; Bio-Rad, Hercules, CA, USA). The cell growth inhibition ratio (\%) at each dose was calculated according to the following formula: $\left[\left(\mathrm{A}_{490}\right.\right.$ of the non-irradiated group - $\mathrm{A}_{490}$ of the irradiated group $) /\left(\mathrm{A}_{490}\right.$ of the non-irradiated group)] x $100 \%$.

Annexin V/propidium iodide (PI) double-staining and flow cytometry of NPC cells. The cells were digested with $0.25 \%$ trypsin (C0201; Beyotime Co., Nantong, China), yielding a single-cell suspension. Cells were washed twice in cold PBS, which was removed from the cell pellet after the second wash. Cells were re-suspended in cold $1 \mathrm{X}$ binding buffer to a concentration of $1 \times 10^{6}$ to $1 \times 10^{7}$ cells $/ \mathrm{ml}$. Next, $10 \mu \mathrm{l}$ of Annexin V-fluorescein isothiocyanate (FITC) $(20 \mu \mathrm{g} / \mathrm{ml}$, $100 \mathrm{~T}$; Abcam, Cambridge, UK) were added to the mixture, gently vortexed, and incubated for $15 \mathrm{~min}$ on ice, with protection from light. Without washing, $10 \mu \mathrm{l}$ of PI $(20 \mu \mathrm{g} / \mathrm{ml}, 100 \mathrm{~T}$; Abcam) was added and incubated for $5 \mathrm{~min}$ on ice, with protection from light. Then, $400 \mu 1$ of binding buffer was added and mixed to achieve a single-cell suspension. After staining, the samples were analyzed using a fluorescence-activated cell sorting (FACS) flow cytometer and CellQuest software (FCS Express V3; BD Biosciences, San Jose, CA, USA). The negative control was a cell sample to which Annexin V-FITC + PI was not added. 


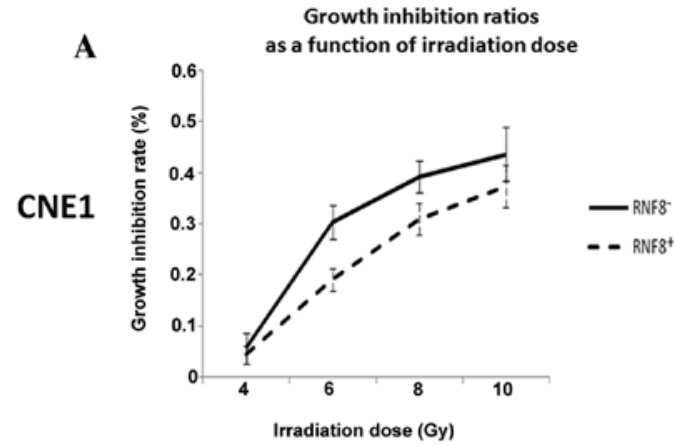

B
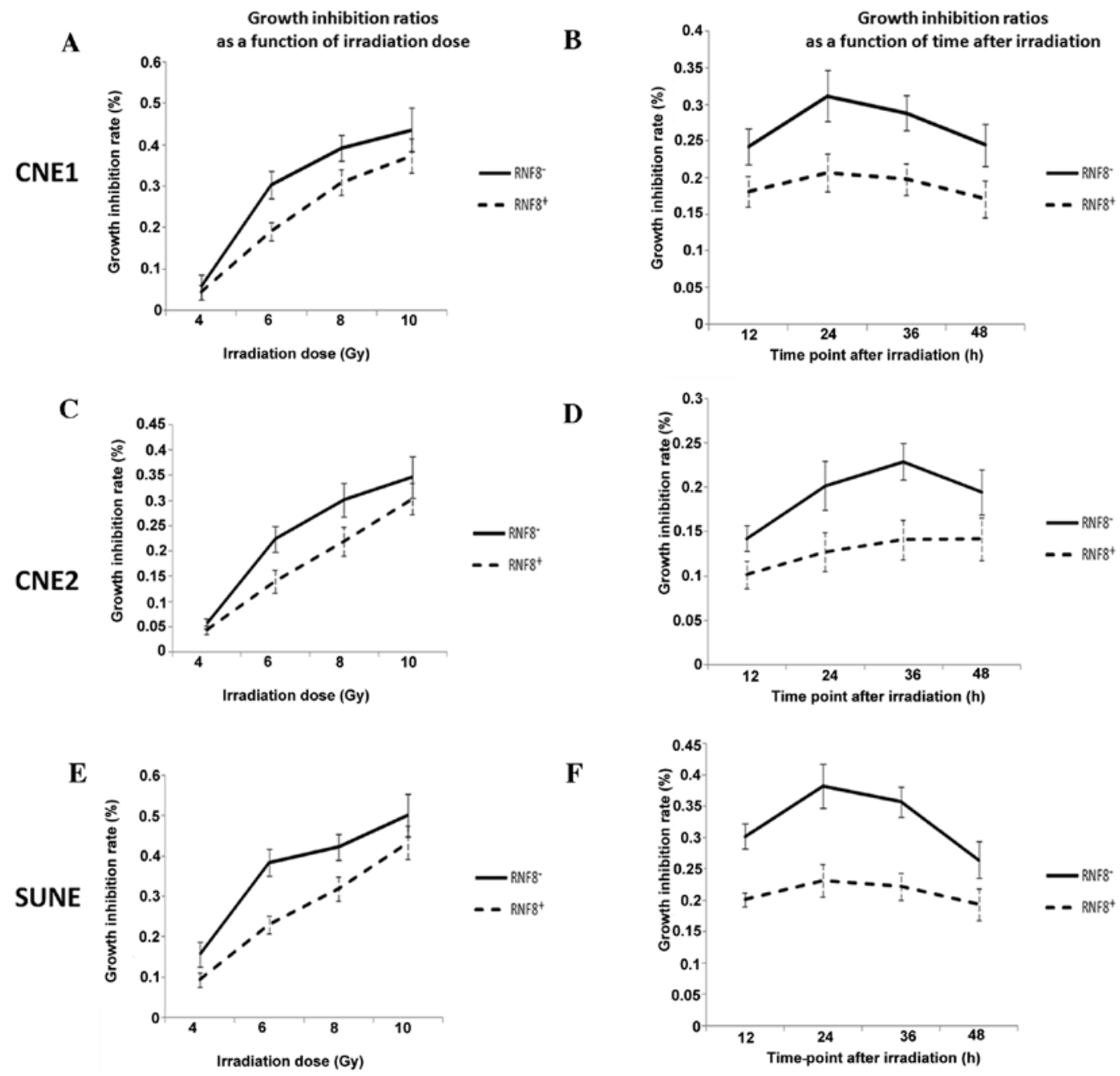

D

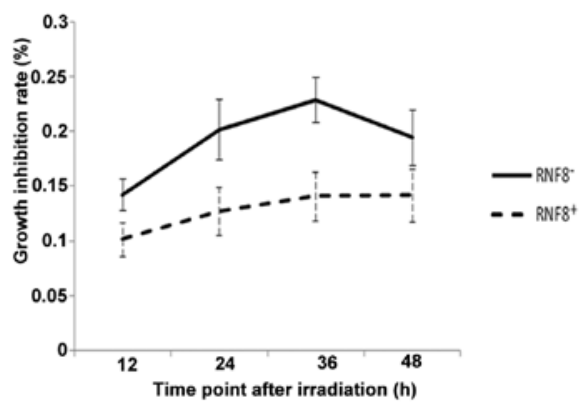

$\mathbf{F}$

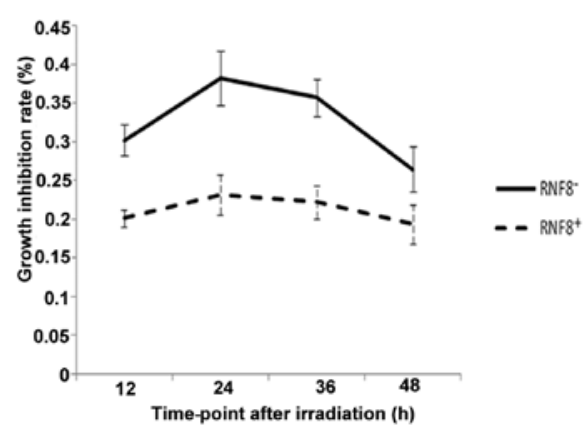

Figure 2. Growth inhibition ratios (\%) of the CNE1 (A and B), CNE2 (C and D) and SUNE (E and F) cell lines as a function of irradiation dose (Gy) and time after irradiation (h), for cells that had been stably transfected (RNF8) or not (RNF8+) with siRNAs against RNF8. RNF8, ring finger protein 8.

Detection of protein expression by flow cytometry. NPC cells were suspended and washed twice with Buffer 1 (PBS containing $0.5 \%$ bovine serum albumin), re-suspended in a fixation and permeabilization solution (B\&D Biosciences Pharmingen, San Jose, CA, USA), and incubated for $20 \mathrm{~min}$. Cells were washed with Buffer 2 (Perm/Wash buffer solution; B\&D Biosciences Pharmingen) and incubated for $4 \mathrm{~h}$ at $4^{\circ} \mathrm{C}$ with the primary antibody, which had been diluted in Buffer 2 . Cells were washed twice with Buffer 2 and re-suspended in $50 \mu \mathrm{l}$ of Buffer 2. DyLight 488-conjugated goat antimouse IgG (Multi Sciences Biotech Co., Beijing, China) was added to the samples, which were incubated in the dark for $30 \mathrm{~min}$ at $4^{\circ} \mathrm{C}$. After staining and washing twice with Buffer 1, the samples were analyzed with a FACS flow cytometer and CellQuest software. Primary antibodies included mouse anti-human monoclonal antibodies for Chk1 and Chk2 (Santa Cruz Biotechnology, Santa Cruz, CA, USA), DNA-PKcs (NeoMarkers Lab Vision Corporation, Fremont, CA, USA), ATM (BioVision Inc., San Francisco, CA, USA), and Ku80 and Nbs1 (Abcam).

Statistical analysis. Data are expressed as the mean \pm standard deviation $(\bar{x} \pm \mathrm{SD})$. All statistical analyses were performed with the SPSS 18.0 software package. Factorial analysis was applied to the measured A values of the MTT assay and to the apoptosis rate at each irradiation dose. Multivariate analysis of variance was applied to the change in the A values and apoptosis rate according to the time after irradiation. One-way analysis of variance and linear correlation were used to analyze the protein levels of DNA-PKcs, Ku80, Nbs1, ATM, Chk1 and Chk2. Differences with a $\mathrm{P}<0.05$ were considered statistically significant.

\section{Results}

Detection of RNF8 protein expression in the RNF8-silenced cells. After repopulating the cells that stably expressed the RNF8-targeting siRNA, we detected RNF8 protein expression with western blot analysis. RNF8 protein expression was significantly downregulated in the RNF8 cells of the three cell lines (Fig. 1).

Growth inhibition ratios. As shown in Fig. 2, the growth inhibition ratio of the CNE1 and SUNE cells increased in an irradiation dose-dependent manner and was highest at $24 \mathrm{~h}$ after irradiation. The growth inhibition ratio of the CNE2 

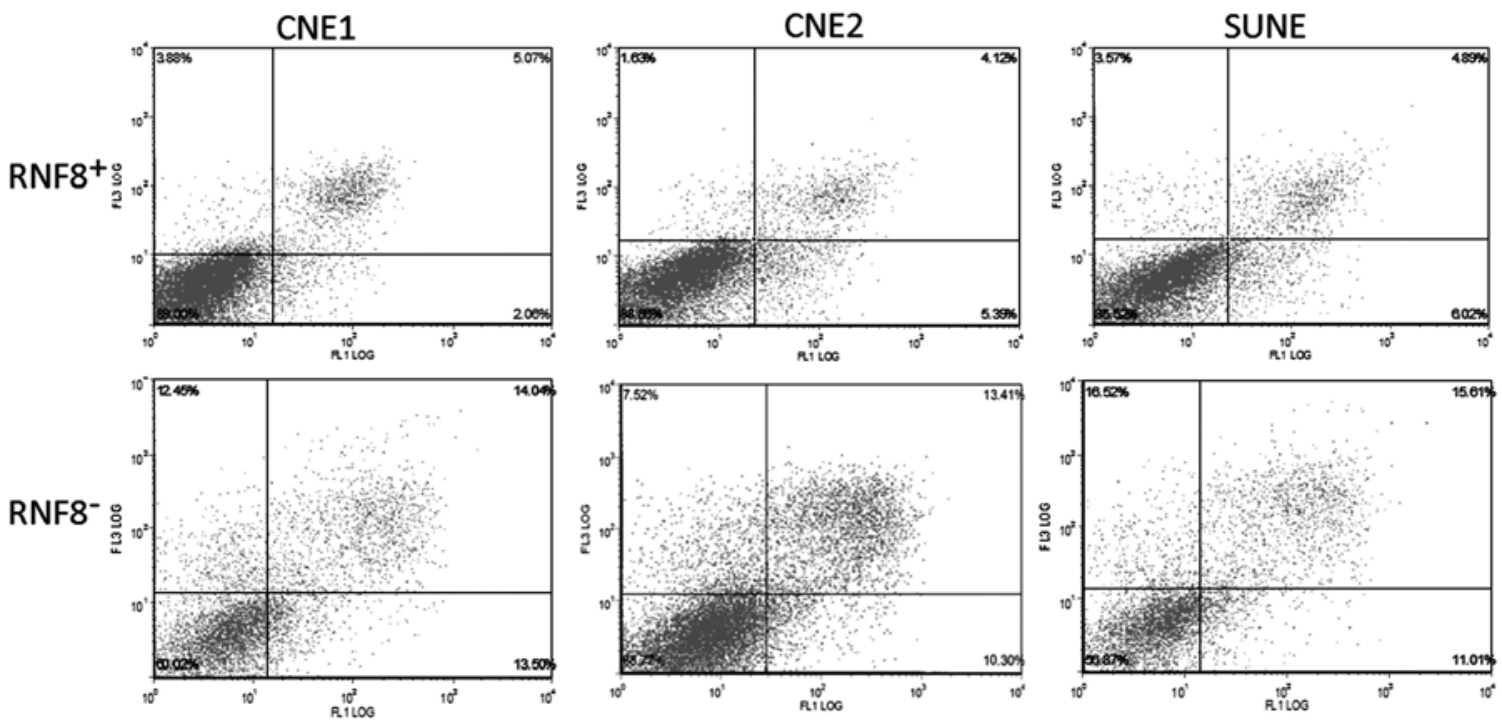

Figure 3. FACS analysis of the percentage of apoptotic cells (\%) among the irradiated CNE1, CNE2 and SUNE cells that were transfected (RNF8) or not $\left(\mathrm{RNF}^{+}\right)$with RNF8-targeting siRNAs. All cell lines were irradiated with $6 \mathrm{~Gy}$. Time-point of analysis was $24 \mathrm{~h}$ after irradiation for the CNE1 and SUNE cells and $36 \mathrm{~h}$ after irradiation for CNE2 cells. Apoptotic cells are indicated in the upper right quadrant of the graphs. RNF8, ring finger protein 8

Table I. Protein expression levels in the irradiated nasopharyngeal cancer cell lines that were transfected or not with siRNAs against RNF8.

\begin{tabular}{|c|c|c|c|c|c|c|}
\hline \multirow[b]{2}{*}{ Protein } & \multicolumn{2}{|c|}{ CNE1 } & \multicolumn{2}{|c|}{ CNE2 } & \multicolumn{2}{|c|}{ SUNE } \\
\hline & $\mathrm{RNF}^{+}$ & RNF8- & $\mathrm{RNF}^{+}$ & RNF8- & $\mathrm{RNF}^{+}$ & RNF8- \\
\hline ATM & $41.2 \pm 5.1$ & $13.1 \pm 2.2$ & $52.3 \pm 10.3$ & $21.7 \pm 4.3$ & $53.6 \pm 9.9$ & $17.2 \pm 3.0$ \\
\hline DNA-PKcs & $35.5 \pm 6.4$ & $28.9 \pm 2.8$ & $37.5 \pm 7.2$ & $31.0 \pm 4.7$ & $33.8 \pm 4.8$ & $27.6 \pm 4.1$ \\
\hline Chk1 & $39.6 \pm 6.9$ & $13.9 \pm 1.9$ & $50.2 \pm 9.8$ & $27.8 \pm 4.5$ & $48.1 \pm 7.4$ & $17.5 \pm 2.3$ \\
\hline Chk2 & $50.0 \pm 9.6$ & $20.3 \pm 4.0$ & $57.9 \pm 11.0$ & $25.6 \pm 3.2$ & $44.2 \pm 6.6$ & $22.4 \pm 3.6$ \\
\hline Nsb1 & $32.7 \pm 5.5$ & $11.2 \pm 1.2$ & $46.8 \pm 5.4$ & $14.1 \pm 2.2$ & $39.4 \pm 5.6$ & $12.9 \pm 1.8$ \\
\hline Ku80 & $23.1 \pm 4.7$ & $31.8 \pm 6.1$ & $35.9 \pm 5.6$ & $40.7 \pm 5.5$ & $33.5 \pm 4.7$ & $38.7 \pm 5.1$ \\
\hline
\end{tabular}

RNF8 cells were not transfected with siRNAs against RNF8. $\mathrm{RNF}^{+}$cells were stably transfected with siRNAs against RNF8. RNF8, ring finger protein 8; ATM, ataxia-telangiectasia mutated.

cells was highest at $36 \mathrm{~h}$ after irradiation. The growth inhibition ratio of RNF8 ${ }^{-}$was higher than that of the $\mathrm{RNF}^{+}$cells in each of the three cell lines. For the 6-Gy dose, the differences between $\mathrm{A} 1$ and $\mathrm{A} 2$, and between $\mathrm{C} 1$ and $\mathrm{C} 2$ were largest after $24 \mathrm{~h}$, whereas the difference between B1 and B2 was largest after $36 \mathrm{~h}$ (Fig. 2).

Apoptotic percentages. As shown in Fig. 3, the percentage of apoptotic cells among the CNE1 and SUNE cells increased in an irradiation dose-dependent manner and was highest $24 \mathrm{~h}$ after irradiation. In the CNE2 cells, the apoptotic percentage was highest $36 \mathrm{~h}$ after irradiation. These results were consistent with the results of the MTT assay. The apoptotic percentage of the RNF8 - cells was higher than that of the RNF8 ${ }^{+}$cells in each of the three cell lines. For the 6-Gy dose, the largest difference was observed between the RNF8 and $\mathrm{RNF}^{+}$cells in the CNE1 and SUNE cell lines after $24 \mathrm{~h}$. In the CNE2 cells, the largest difference for the 6-Gy dose occurred after 36 h (Fig. 3).
Protein expression levels. Expression levels of the Chk1, Chk2, ATM and Nbs1 proteins in the RNF8 cells in each of the three cell lines were lower than the corresponding levels in the $\mathrm{RNF}^{+}$cells after irradiation $(\mathrm{P}<0.01)$. The expression of DNA-PKcs was not different between the RNF8 and $\mathrm{RNF}^{+}$cells. The expression of Ku80 was higher in RNF8 cells compared to the RNF8 ${ }^{+}$cells, but the difference was not significant (Fig. 4, Table I).

\section{Discussion}

The results of the present study indicate that RNF8 recruits and ubiquitinates many factors, including Chk1, Chk2, DNA-PKcs, ATM and Nbs1, to repair DNA damage, including DSBs. This mechanism leads to the radioresistance of NPC cells.

Lu et al (9) previously reported that RNF8 directly binds to and ubiquitinates Nbs1, and that RNF8 ubiquitination activity is needed for the localization of Nbs1 to DSBs. Further analysis revealed that the RNF8 ubiquitination of Nbs1 is 
A

ATM
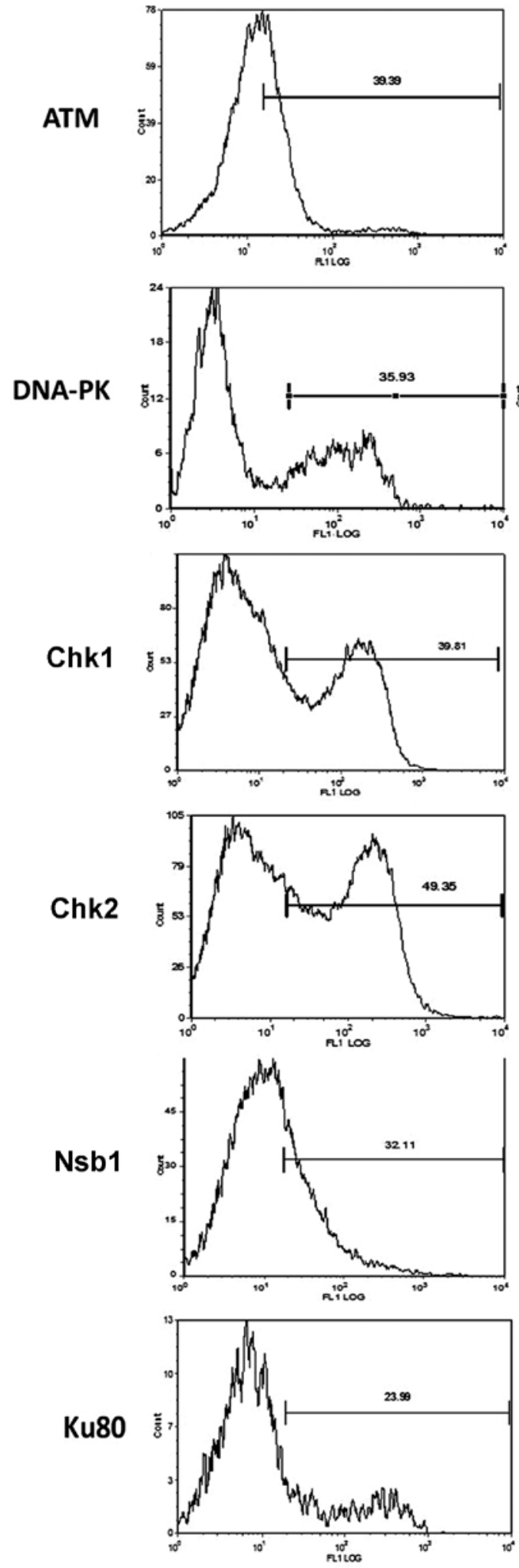

RNF8-
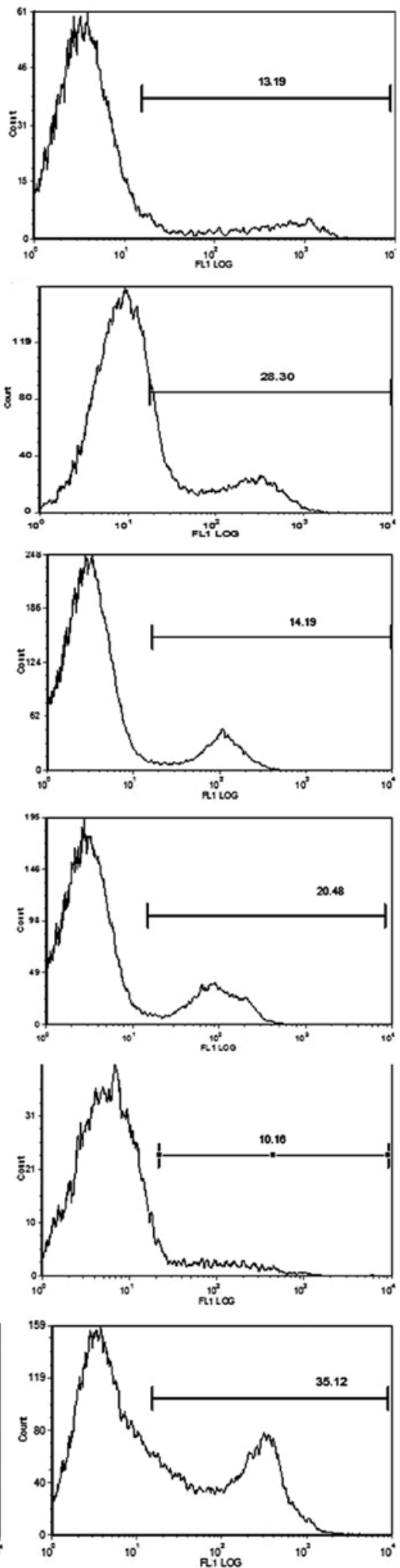

Figure 4. (A) Protein expression levels of ATM, DNA-PK, Chk1, Chk2, Nbs1 and Ku80 in the irradiated CNE1 cells that were transfected (RNF8-) or not $\left(\mathrm{RNF}^{+}\right)$with siRNAs against RNF8 $24 \mathrm{~h}$ after 6-Gy irradiation. The x-axes show fluorescence intensity, the y-axes show cell counts and the numbers on the graphs indicate the percentage of positive cells. RNF8. RNF8, ring finger protein 8; ATM, ataxia-telangiectasia mutated.

important for promoting DSB repair by HR. The HR process requires the function of the MRN complex, which recognizes the lesions and recruits ATM to DSBs (33). Thereafter, a series of proteins are activated to repair DNA. In the present study, the expression of Nbs1 increased after radiation in the RNF8 ${ }^{+}$ cells, but not in the RNF8- cells. This result and the previous 
B
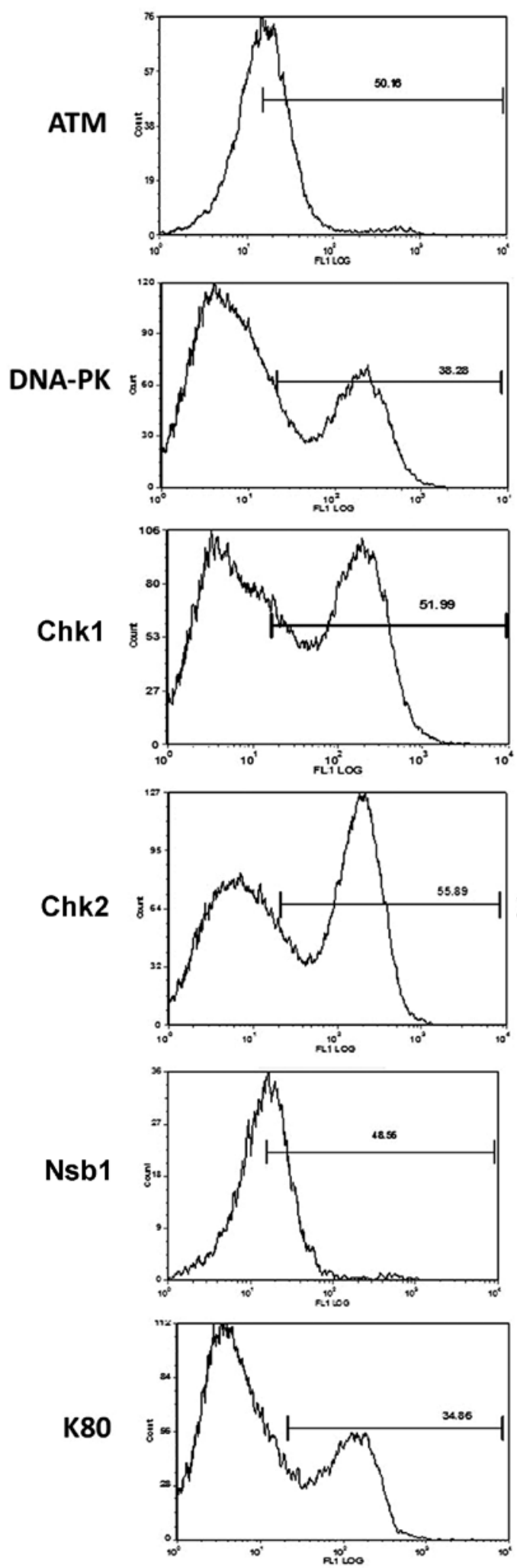

RNF8-
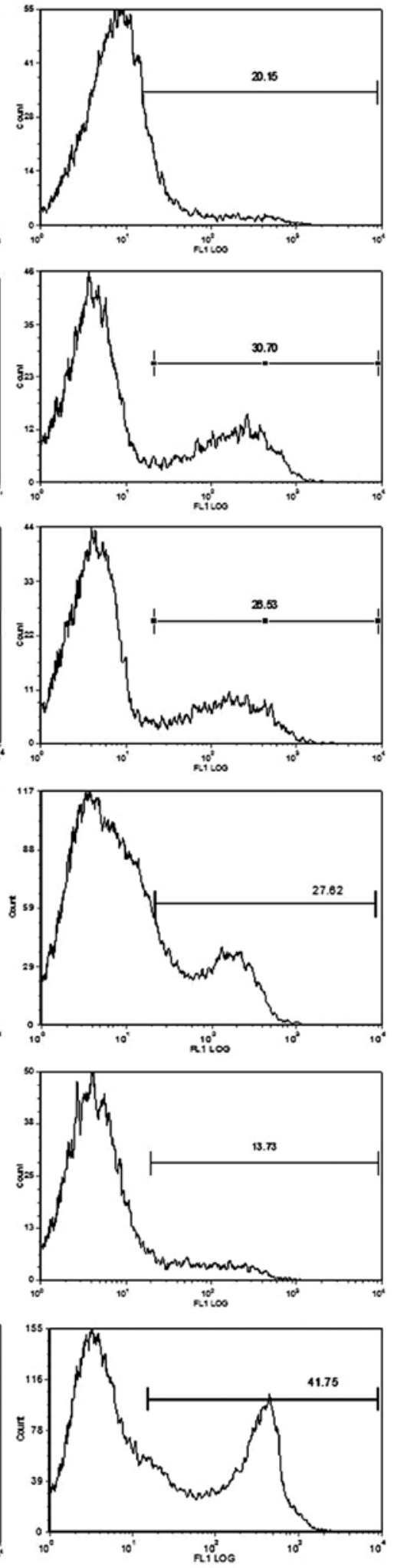

Figure 4. Continued. (B) Protein expression levels of ATM, DNA-PK, Chk1, Chk2, Nbs1 and Ku80 in the irradiated CNE2 cells that were transfected (RNF8-) or not $\left(\mathrm{RNF}^{+}\right)$with siRNAs against RNF8 $36 \mathrm{~h}$ after 6-Gy irradiation. The x-axes show fluorescence intensity, the y-axes show cell counts and the numbers on the graphs indicate the percentage of positive cells. RNF8. RNF8, ring finger protein 8; ATM, ataxia-telangiectasia mutated.

observations indicate that RNF8 ubiquitinates and activates Nsb1, which is important to the HR pathway and repairs the DNA.
Ku80 forms a ring-like structure and completely encircles the DSB ends. The ring-like structure may be translocated or removed to provide room for DNA-PKcs and other repair 
C

ATM
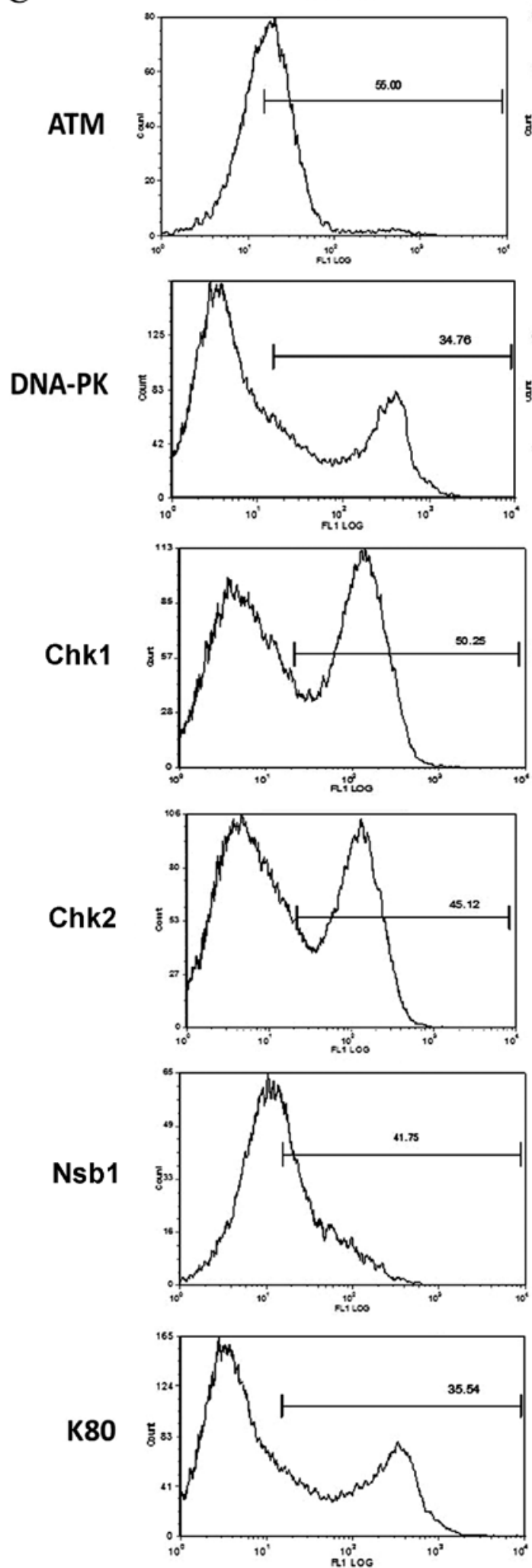

RNF8-
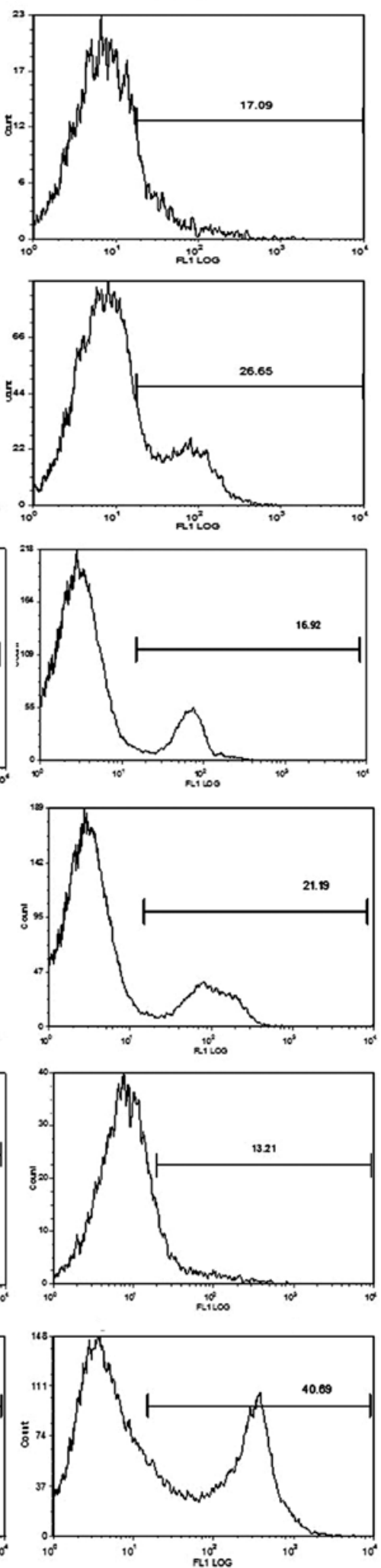

Figure 4. Continued. (C) Protein expression levels of ATM, DNA-PK, Chk1, Chk2, Nbs1 and Ku80 in the irradiated SUNE cells that were transfected (RNF8-) or not $\left(\mathrm{RNF}^{+}\right)$with siRNAs against RNF8 $24 \mathrm{~h}$ after 6-Gy irradiation. The x-axes show fluorescence intensity, the y-axes show cell counts and the numbers on the graphs indicate the percentage of positive cells. RNF8. RNF8, ring finger protein 8; ATM, ataxia-telangiectasia mutated.

factors (34). RNF8 may specifically target Ku80 degradation or removal at DSB sites. Degradation of Ku80 by RNF8 is required for the removal of Ku80 from DSBs (10).
After radiation, the growth inhibition ratio of RNF8- NPC cells was higher than that of $\mathrm{RNF}^{+}$cells. Thus, cells in which RNF8 was silenced were more susceptible to radiation 


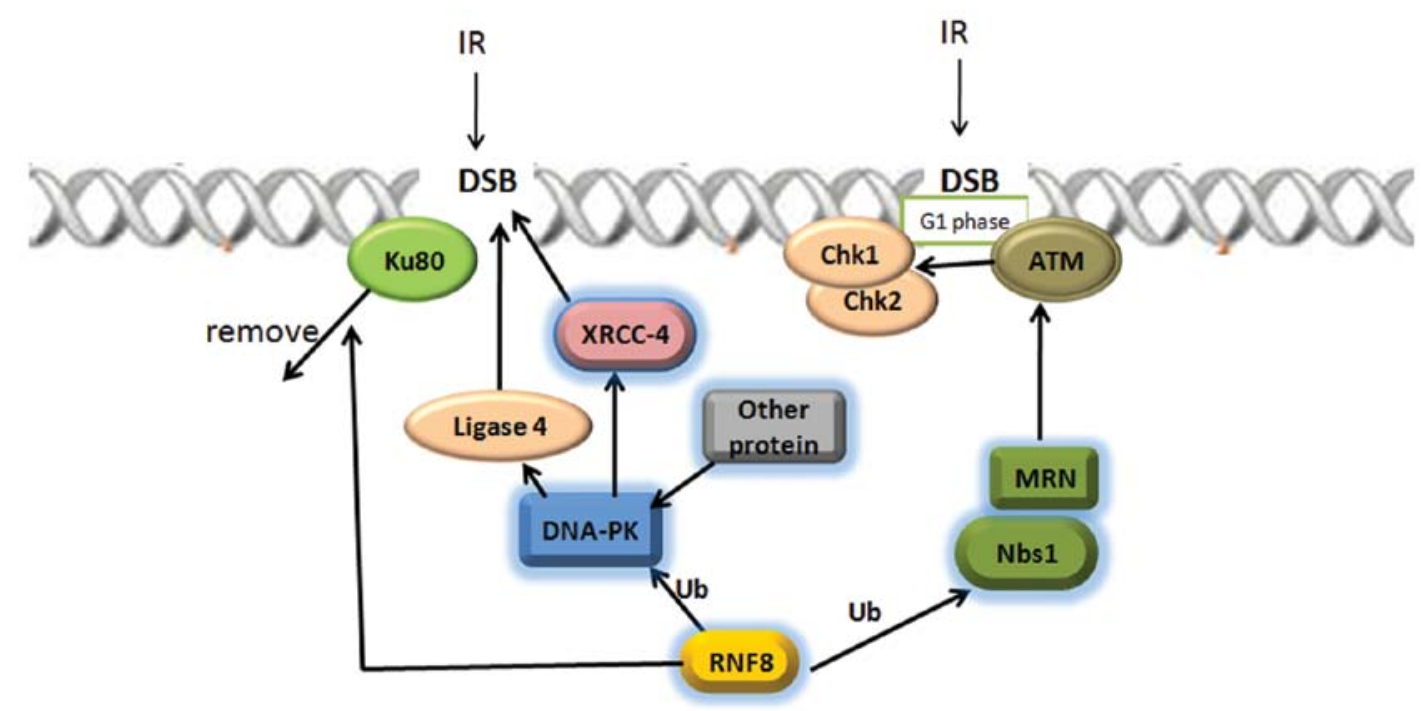

Figure 5. The mechanism of RNF8 in the radioresistance of NPC cells is portrayed as a schematic diagram. RNF8. RNF8, ring finger protein 8; NPC, nasopharyngeal cancer.

damage. The flow cytometric results showed that, after radiation, the apoptosis rate of the RNF8- cells was increased, but the apoptosis rate of the $\mathrm{RNF} 8^{+}$cells was not changed. This result was consistent with the MTT assay results. The apoptosis rate of CNE2 cells was lower than the apoptosis rates of the CNE1 and SUNE cells, indicating that CNE2 cells have stronger radioresistance ability.

We detected the expression of Chk1, Chk2, DNA-PKcs, ATM, Ku80 and Nbs1 in the CNE1, CNE2 and SUNE cells before and after radiation. The FACS results showed that before radiation, each protein had a similar expression level in the $\mathrm{RNF}^{-}$and $\mathrm{RNF}^{+}$cells. After radiation, the Chk1, Chk2, DNA-PKcs, ATM and Nbs1 protein levels increased in the three cell lines in the RNF8 ${ }^{+}$cells, but the level of Ku80 decreased. In the RNF8- cells, only the DNA-PKcs and Ku80 protein expression levels increased. In all three cell lines after irradiation, the expression levels of ATM, Chk1, Chk2 and Nbs1 in the RNF8 cells were lower than the expression levels in the RNF8+ cells. The expression of DNA-PKcs almost did not change, but the level of Ku80 was slightly higher.

We conjecture that the HR pathway through which ATM acts mainly received ubiquitinated regulation by RNF8. In contrast, the NHEJ pathway through which DNA-PK acts was not only affected by RNF8, but also regulated in other ways. In the RNF8 cells, the decrease in KU80 degradation by RNF8 resulted in prolonged retention of KU80 at sites of damage and impaired NHEJ repair. The observation of higher protein expression levels of Chk1, Chk2, DNA-PKcs, ATM and Nbs1 in CNE2 compared to the CNE1 and SUNE cells provides further evidence that CNE2 cells have a stronger radioresistance ability than the other two cell lines.

The mechanism by which RNF8 improves the radioresistance of NPC cells may be theorized from the above experimental results. Irradiation leads to an increased expression of RNF8, which then ubiquitinates DNA-PKcs, ATM, and some other proteins to repair the irradiated DNA. Both NHEJ and HR are involved in DNA repair. DNA-PK activates $\mathrm{XRCC} 4$, ligase 4 and other factors. ATM triggers the check- point between the $\mathrm{G} 1$ and $\mathrm{S}$ phases, and activates Chk1 and Chk2 to repair the injured DNA. Nsb1 is recruited to the DSB site and is ubiquitinated. Nsb1 promotes DNA repair through the HR pathway. Overall, the DNA damage response prevents cells from undergoing apoptosis. The NHEJ pathway is also regulated by other factors, which have not yet been studied.

In conclusion, RNF8 recruits and ubiquitinates many factors, including Chk1, Chk2, DNA-PKcs, ATM and Nbs1, to the impaired DNA site. Ku80 is removed from the impaired site to repair the DNA damage (Fig. 5). This mechanism leads NPC cells to acquire radioresistance.

\section{Acknowledgements}

We thank Medjaden Bioscience Limited for assisting in the preparation of this manuscript.

\section{References}

1. Xie SH, Yu IT, Tse LA, Mang OW and Yue L: Sex difference in the incidence of nasopharyngeal carcinoma in Hong Kong 1983-2008: suggestion of a potential protective role of oestrogen. Eur J Cancer 49: 150-155, 2013.

2. Raghupathy R, Hui EP and Chan AT: Epstein-Barr virus as a paradigm in nasopharyngeal cancer: from lab to clinic. Am Soc Clin Oncol Educ Book 34: 149-153, 2014.

3. Siti-Azrin AH, Norsa'adah B and Naing NN: Five-year survival and median survival time of nasopharyngeal carcinoma in Hospital Universiti Sains Malaysia. Asian Pac J Cancer Prev 15: 6455-6459, 2014.

4. Jardel P, Thariat J, Blanchard P, Elloumi F, Toumi N, Bensadoun RJ, Frikha M, Daoud J and Bourhis J: Nasopharyngeal cancers, an overview. Bull Cancer 101: 445-454, 2014 (In French).

5. Hamilton SN, Ho C, Laskin J, Zhai Y, Mak P and Wu J: Asian versus non-Asian outcomes in nasopharyngeal carcinoma: a North American population-based analysis. Am J Clin Oncol: May 29, 2014 (Epub ahead of print). doi: 10.1097/ COC.0000000000000091.

6. Zeng L, Tian YM, Sun XM, Huang Y, Chen CY, Han F, Liu S, Lan M, Guan Y, Deng XW, et al: Intensity-modulated radiotherapy for stage IVA/IVB nasopharyngeal carcinoma: clinical outcomes and patterns of failure in an endemic area in China. Strahlenther Onkol 190: 993-1000, 2014. 
7. Dou H,Hu D, Lam C, Liu Y, Wang X and Zhang W: Retrospective analysis of results of treatment for nasopharyngeal carcinoma in Macao. Chin J Cancer Res 26: 148-158, 2014.

8. Ueno T, Endo K, Kondo S, Wakisaka N, Murono S, Ito M and Yoshizaki T: Factors affecting outcomes of alternating chemoradiotherapy for nasopharyngeal cancer. Ann Otol Rhinol Laryngol 123: 509-516, 2014.

9. Lu CS, Truong LN, Aslanian A, Shi LZ, Li Y, Hwang PY, Koh KH, Hunter T, Yates JR III, Berns MW, et al: The RING finger protein RNF8 ubiquitinates Nbs1 to promote DNA double-strand break repair by homologous recombination. J Biol Chem 287: 43984-43994, 2012.

10. Feng L and Chen J: The E3 ligase RNF8 regulates KU80 removal and NHEJ repair. Nat Struct Mol Biol 19: 201-206, 2012.

11. Wu J, Chen Y, Lu LY, Wu Y, Paulsen MT, Ljungman M, Ferguson DO and $\mathrm{Yu} \mathrm{X}$ : Chfr and RNF8 synergistically regulate ATM activation. Nat Struct Mol Biol 18: 761-768, 2011

12. Pinder JB, Attwood KM and Dellaire G: Reading, writing, and repair: the role of ubiquitin and the ubiquitin-like proteins in DNA damage signaling and repair. Front Genet 4: 45, 2013.

13. Mailand N, Bekker-Jensen S, Faustrup H, Melander F, Bartek J, Lukas C and Lukas J: RNF8 ubiquitylates histones at DNA double-strand breaks and promotes assembly of repair proteins. Cell 131: 887-900, 2007.

14. Huen MS, Grant R, Manke I, Minn K, Yu X, Yaffe MB and Chen J: RNF8 transduces the DNA-damage signal via histone ubiquitylation and checkpoint protein assembly. Cell 131: 901-914, 2007.

15. Kolas NK, Chapman JR, Nakada S, Ylanko J, Chahwan R, Sweeney FD, Panier S, Mendez M, Wildenhain J, Thomson TM, et al: Orchestration of the DNA-damage response by the RNF8 ubiquitin ligase. Science 318: 1637-1640, 2007

16. Wang B and Elledge SJ: Ubc13/Rnf8 ubiquitin ligases control foci formation of the Rap80/Abraxas/Brca1/Brcc36 complex in response to DNA damage. Proc Natl Acad Sci USA 104: 20759-20763, 2007.

17. Ito K, Adachi S, Iwakami R, Yasuda H, Muto Y, Seki N and Okano Y: N-Terminally extended human ubiquitin-conjugating enzymes (E2s) mediate the ubiquitination of RING-finger proteins, ARA54 and RNF8. Eur J Biochem 268: 2725-2732, 2001.

18. Bartocci $C$ and Denchi EL: Put a RING on it: regulation and inhibition of RNF8 and RNF168 RING finger E3 ligases at DNA damage sites. Front Genet 4: 128, 2013.

19. Zhou H, Mu X, Chen J, Liu H, Shi W, Xing E, Yang K and Wu G: RNAi silencing targeting RNF8 enhances radiosensitivity of a non-small cell lung cancer cell line A549. Int J Radiat Biol 89: $708-715,2013$

20. Symington LS and Gautier J: Double-strand break end resection and repair pathway choice. Annu Rev Genet 45: 247-271, 2011.
21. Santivasi WL and Xia F: Ionizing radiation-induced DNA damage, response, and repair. Antioxid Redox Signal 21: 251-259, 2014.

22. Takahashi A, Kubo M, Ma H, Nakagawa A, Yoshida Y, Isono M, Kanai T, Ohno T, Furusawa Y,Funayama T, et al: Nonhomologous end-joining repair plays a more important role than homologous recombination repair in defining radiosensitivity after exposure to high-LET radiation. Radiat Res 182: 338-344, 2014.

23. Keijzers G, Maynard S, Shamanna RA, Rasmussen LJ, Croteau DL and Bohr VA: The role of RecQ helicases in non-homologous end-joining. Crit Rev Biochem Mol Biol 49: 463-472, 2014

24. Taleei $\mathrm{R}$ and Nikjoo $\mathrm{H}$ : The non-homologous end-joining (NHEJ) pathway for the repair of DNA double-strand breaks: I. a mathematical model. Radiat Res 179: 530-539, 2013

25. Goodwin JF and Knudsen KE: Beyond DNA repair: DNA-PK function in cancer. Cancer Discov 4: 1126-1139, 2014

26. Truong LN, Li Y, Sun E, Ang K, Hwang PY and Wu X: Homologous recombination is a primary pathway to repair DNA double-strand breaks generated during DNA rereplication. J Biol Chem 289: 28910-28923, 2014.

27. Lim YC, Roberts TL, Day BW, Stringer BW, Kozlov S, Fazry S, Bruce ZC, Ensbey KS, Walker DG, Boyd AW, et al: Increased sensitivity to ionizing radiation by targeting the homologous recombination pathway in glioma initiating cells. Mol Oncol 8: 1603-1615, 2014.

28. Liu T and Huang J: Quality control of homologous recombination. Cell Mol Life Sci 71: 3779-3797, 2014.

29. Daley JM, Kwon Y, Niu H and Sung P: Investigations of homologous recombination pathways and their regulation. Yale J Biol Med 86: 453-461, 2013.

30. Tomita M: Involvement of DNA-PK and ATM in radiation- and heat-induced DNA damage recognition and apoptotic cell death. J Radiat Res (Tokyo) 51: 493-501, 2010.

31. Dobbs TA, Tainer JA and Lees-Miller SP: A structural model for regulation of NHEJ by DNA-PKcs autophosphorylation. DNA Repair (Amst) 9: 1307-1314, 2010.

32. Bekker-Jensen S and Mailand N: Ubiquitin and the DNA damage response: A new handle on histones. Cell Cycle 11: 3153, 2012.

33. Zhou Y and Paull TT: DNA-dependent protein kinase regulates DNA end resection in concert with Mre11-Rad50-Nbs1 (MRN) and ataxia telangiectasia-mutated (ATM). J Biol Chem 288: 37112-37125, 2013.

34. Alshareeda AT, Negm OH, Albarakati N, Green AR, Nolan C, Sultana R, Madhusudan S, Benhasouna A, Tighe P, Ellis IO, et al: Clinicopathological significance of KU70/KU80, a key DNA damage repair protein in breast cancer. Breast Cancer Res Treat 139: 301-310, 2013. 